Communications in Physics, Vol. 31, No. 2 (2021), pp. 227-234

DOI:10.15625/0868-3166/15674

\title{
ANISOTROPIC MAGNETORESISTANCE EFFECT OF NICKEL NANOWIRES
}

\author{
PHAM DO CHUNG $^{1, \dagger}$, NGO VAN THANH ${ }^{2}$ AND JEAN-ERIC WEGROWE ${ }^{3}$ \\ ${ }^{1}$ Hanoi National University of Education, \\ 136 Xuan Thuy, Cau Giay, Hanoi, Vietnam \\ ${ }^{2}$ Centre for Informatics and Computing, \\ Vietnam Academy of Science and Technology, \\ 18 Hoang Quoc Viet, Nghia Do, Cau Giay, Hanoi, Vietnam \\ ${ }^{3}$ LSI, Ecole Polytechnique, \\ CEA/DRF/IRAMIS, CNRS, Institut Polytechnique de Paris, \\ F-91128 Palaiseau, France
}

E-mail: ${ }^{\dagger}$ chungpd@hnue.edu.vn

Received 10 November 2020

Accepted for publication 15 December 2020

Published 15 January 2020

\begin{abstract}
In this work, we study the magnetic properties of nickel nanowires by measuring their anisotropic magnetoresistance at room temperature. The single nickel nanowire is grown by electrodeposition in a polymer membrane (Polycarbonate). We measure the anisotropic magnetoresistance effect of nickel nanowires for the various values of the magnitudes and orientations of an external magnetic field. The results clearly show the existence the anisotropic magnetoresistance effect in the nickel nanowires. Besides, the experimental data are best fit to the analytical calculations using the Stoner-Wohlfarth model for the magnetization of the wires.
\end{abstract}

Keywords: AMR, single domain, magnetic nanowire.

Classification numbers: 75.47.De, 81.07.Gf, 75.30.Gw, 85.75.-d. 


\section{INTRODUCTION}

Spintronics is one of the prominent fields in finding out a new phenomenon that was discovered in the past few decades. In solid-state devices, ones have found the dependence of the electron transport on the spin which is an intrinsic property of electron. So, the concept of spintronics relates to spin-charge coupling in metallic materials. The performance of spintronic devices is controllable therefore it provides a larger diversity of functionality. Spintronics has become the new science of computers and memory chips in the good advantages to reduce their power consumption, to increase their memory and processing capabilities [1,2].

Giant magnetoresistance (GMR) is one of typical phenomena in spintronics that is a quantum effect in essence. GMR effect was found in the multilayer materials with the electron scattering depended on the spin orientation. The tunnelling magnetoresistance (TMR) effect was observed in a tunnel junction composed of two ferromagnetic layers and a thin insulator one [3-5]. Besides, the anisotropic magnetoresistance (AMR) effect is a most interesting phenomenon in ferromagnetic materials. The electrical resistance of materials depends on the relative angle between the direction of magnetization and the one of electric current [6-9]. The AMR effect occurs not only in bulk materials [10-12] but also in nanomaterials such as the ferromagnetic nanowires (NWs) [11-13]. The magnetoresistance of NWs is easily in control of the shape and the size of wires for making the magnetic devices such as memory, sensor, signal processing, logic $[10-12,14]$.

Recently, Nickel NWs materials have been extensively studied in both experimental measurements and theoretical calculations. Because the susceptibility is very small in Ni NWs comparing to in other ferromagnetic materials, the activity of these devices could be controlled by a weak external magnetic field for saving the power consumption. However, the difficulty in experiments is that the magnetization of a single nickel nanowire is extremely small (less than $10 \mathrm{emu}$ in the nano-scale of size) $[7,8,12,15]$. So, ones have to use the micro-SQUID susceptor-meters or the magnetic force microscopy (MFM) probes which are the high cost equipment $[7,8,16]$.

In this paper, we presented the experimental measurement of the resistance of the nickel nanowires at room temperature. The samples were made by the electrodeposition technique with the size of $10 \mathrm{~nm}, 30 \mathrm{~nm}$ and $100 \mathrm{~nm}$. We investigated the AMR effect by varying the applied magnetic field on both magnitude and direction. The results were confirmed by fitting the experimental data to the theoretical calculations based on the Stoner-Wohlfarth model.

\section{EXPERIMENT}

\section{II.1. Materials}

In this study, the electrodeposition technique is used to produce the nickel nanowires that is one of common methods to prepare the nanostructured samples $[8,10,15,17]$. The structure of polycarbonate (PC) membrane filter is shown in Fig. 1, the membranes which have the cylindrical pores inside with the diameter in the range of $10 \mathrm{~nm}$ to $100 \mathrm{~nm}$, are about $6 \mu \mathrm{m}$ of thickness. The two gold layers at surfaces are deposited by the sputtering technique to prevent the blocking of the pores. The below layer is a solid base of $200 \mathrm{~nm}$ thickness, and the upper one of $35 \mathrm{~nm}$ has the pores coinciding exactly with the cylindrical ones of the membrane. The $\mathrm{PC}$ membrane is soaked in the aqueous electrolytic solution composed of $\mathrm{NiSO}_{4}$ and $\mathrm{H}_{3} \mathrm{BO}_{4}$ at $\mathrm{pH}=6.2$. During the electrochemical action, $\mathrm{Ni}$ nanowires is growing up in the cylindrical pores of the membrane, 
when one of them is filled, the current through the two electrodes (gold layers) is soaring from zero to high intensity and then we can terminate the sample production process $[8,18,19]$.

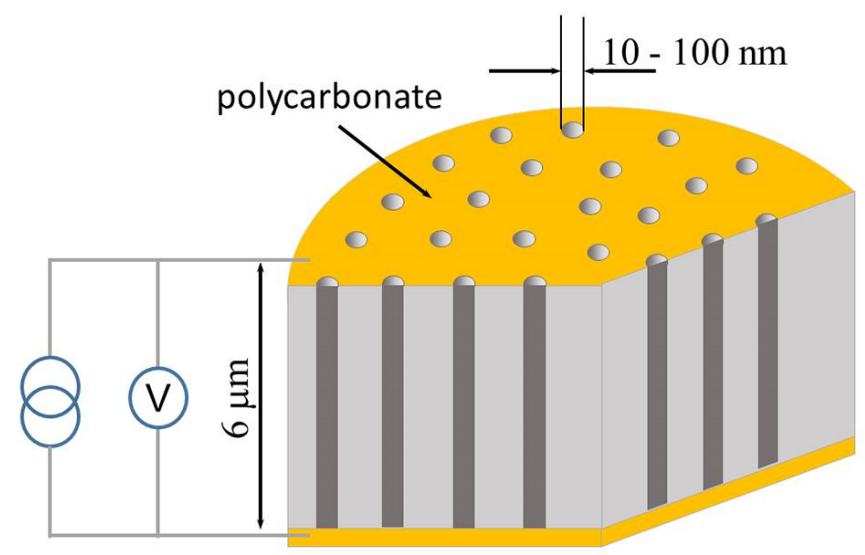

Fig. 1. The structure of polycarbonate membrane filter.
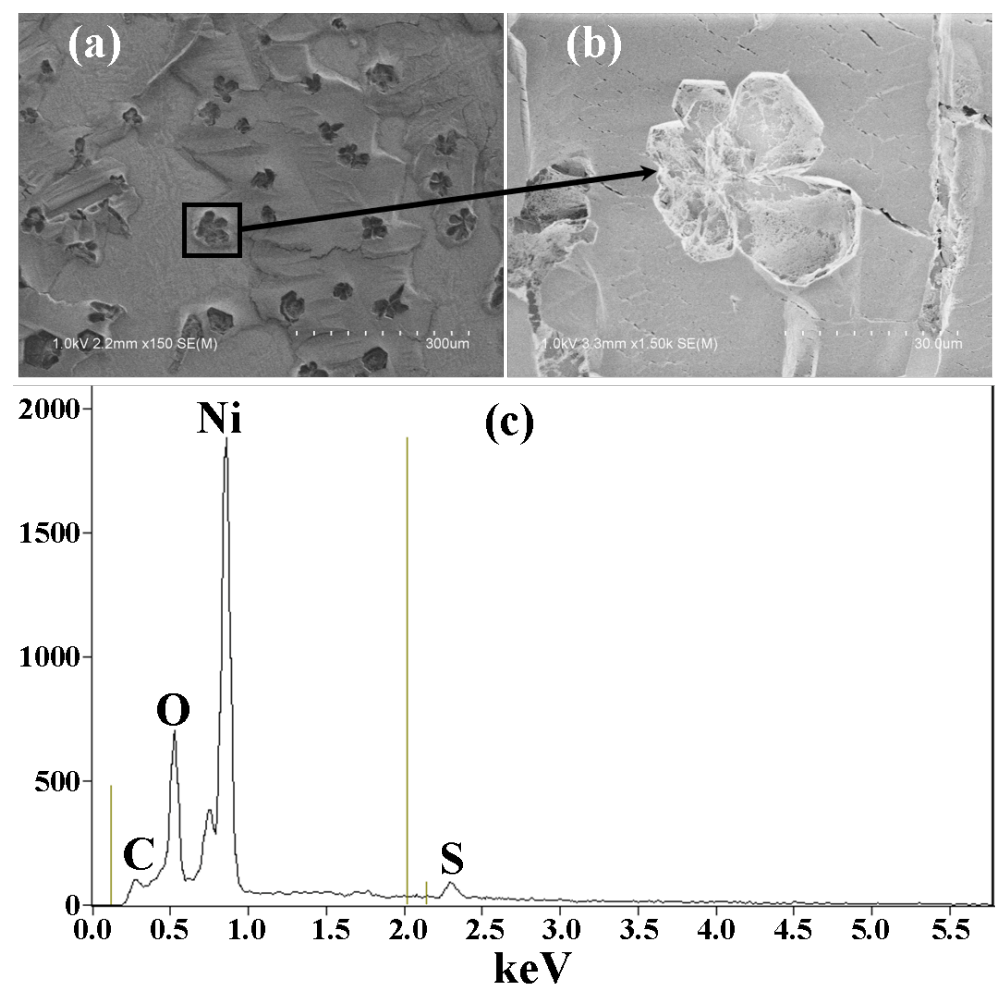

Fig. 2. (a) SEM image of Ni NWs, (b) SEM image of a single NW with the diameter of $30 \mathrm{~nm},(\mathrm{c})$ the EDS spectrum. 
We obtained here the samples notated by PC10, PC30 and PC100 for the Ni NWs with the diameters of $10 \mathrm{~nm}, 30 \mathrm{~nm}$ and $100 \mathrm{~nm}$, respectively. SEM images of PC30 are shown in the figures $2 \mathrm{a}$ and $2 \mathrm{~b}$ (taken by FESEM equipment named Hitachi S-4800 at LSI, Ecole Polytechnique, French). Fig. $2 \mathrm{c}$ is the energy dispersive X-ray spectroscopy (EDS) spectrum of nanowire which shows the contributions of $\mathrm{C}(11 \%), \mathrm{O}(16 \%), \mathrm{S}(1 \%)$ and $\mathrm{Ni}(72 \%)$.

\section{II.2. Experimental methods}

We designed an experimental measuring system with the components are shown in Fig. 3a. The sample was mounted in a self-constructed holder connecting to the amperemeter and voltmeter. The holder was put in the Faraday cage to reduces the electromagnetic noise. We used the Keithley 6221 device to provide a fixed DC current $I<100 \mu \mathrm{A}$ across the nanowire axis in $z$ direction. The Keithley 2182A nanovoltmeter device was used for measuring voltages between the ends of nanowire. Note that, this device has just $15 \mathrm{nV}$ noise at $1 \mathrm{~s}$ response time with an external circuit of $1 \mathrm{k} \Omega$ resistance.

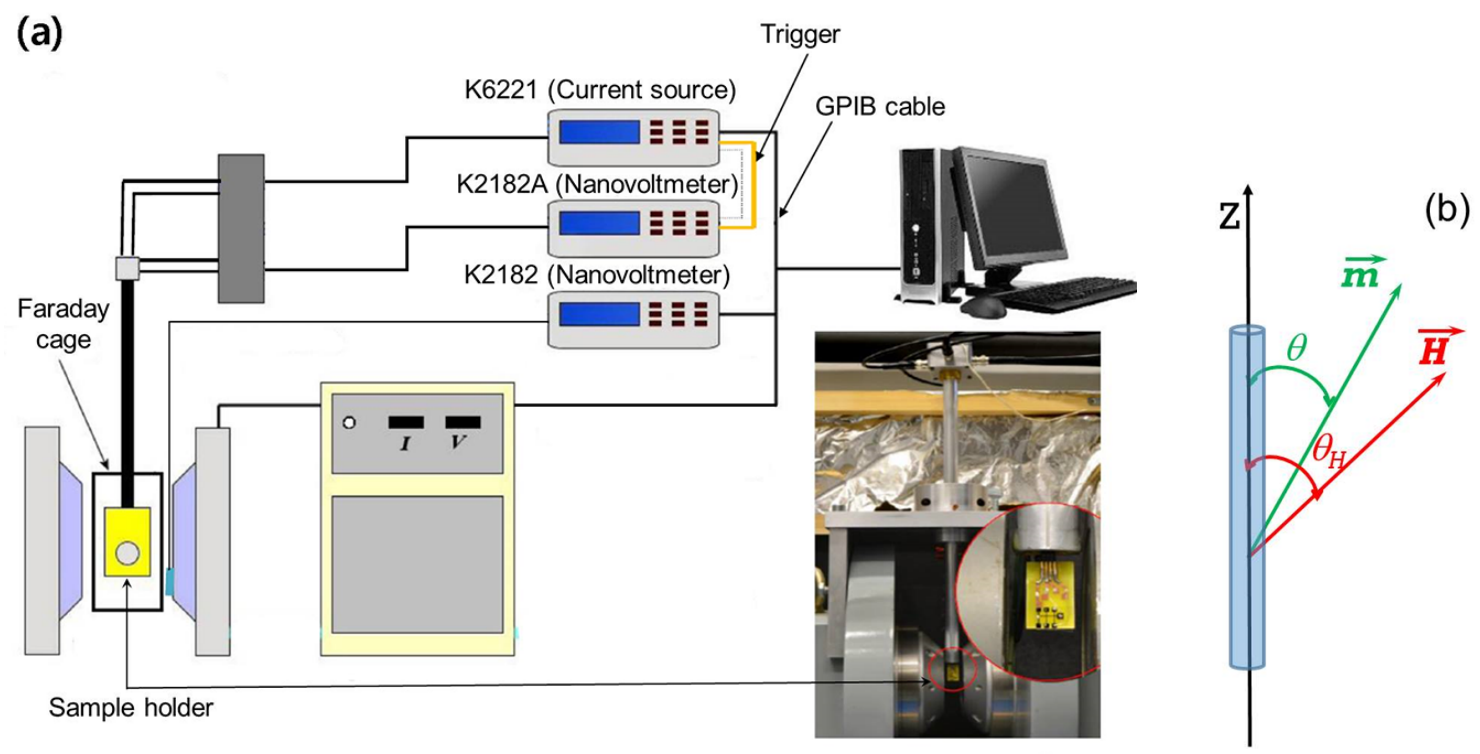

Fig. 3. (a) diagram of the experimental measuring system, (b) the angles between the magnetic field and the $z$ direction.

In this experiment, we applied an external magnetic field $\mathbf{H}$ in a direction which made an angle $\theta_{H}$ with the nanowire axis (see in Fig. 3b). This field was provided by the Bouhnik electromagnet and the current was supplied by a $\pm 50 \mathrm{~A}$ generator. We measured the voltage across the nanowire at various values of $H$ and $\theta_{H}$, then the resistance $R$ of the nanowire was easily derived from Ohm's law. We used here the expression of AMR ratio $[1,5-7,18,19]$ as

$$
\left(\frac{\Delta R}{R}\right)=\frac{R-R_{\min }}{R_{\min }}
$$

with $R_{\min }$ is the minimum resistance of the nanowire measured at $\theta_{H}=90^{\circ}$ where the applied field is perpendicular to the wire axis ( $H \perp z$ in Fig. 3b). 
For a macroscopic scale sample, the nickel NWs can be considered as a single domain so that their magnetization is almost uniform along, i.e., $\mathbf{m}=m_{s} \mathbf{e}_{u}$, in which $m_{s}$ is their saturation magnetization and $\mathbf{e}_{u}$ is the unit vector of modulus $m_{s}$. Let denote $\theta$ the angle between $\mathbf{m}$ and the $z$ axis obtained by minimising the ferromagnetic free energy in the Stoner-Wohlfarth model

$$
E(\theta)=-H m_{s} \cos \left(\theta_{H}-\theta\right)-H_{d} m_{s} \cos ^{2} \theta
$$

where $H_{d}$ is the demagnetising field. The relation between the estimated resistance and the magnetization is given by

$$
R(m)=R_{\min }+\delta R \cos ^{2} \theta
$$

In which, $\delta R=R_{\max }-R_{\min }$ is the maximum variation of resistance due to AMR, $R_{\max }$ is the maximum resistance of the nanowire measured at the applied field $\theta_{H}=0^{\circ}$.

We defined a similar formula as the equation (1) for the AMR ratio of estimated resistance:

$$
\begin{aligned}
\left(\frac{\Delta R}{R}\right) & =\frac{R(m)-R_{\min }}{R_{\min }} \\
& =\frac{\delta R}{R_{\min }} \cos ^{2} \theta,
\end{aligned}
$$

where $\theta$ is obtained by solving the equation

$$
\frac{\partial E(\theta)}{\partial \theta}=0
$$

\section{RESULTS AND DISCUSSIONS}

In this section, we show the measured data and the analytical results for three samples PC10, PC30 and PC100. The external magnetic field $\mathrm{H}$ is varying from $-14 \mathrm{kOe}$ to $+14 \mathrm{kOe}$ with some angles $\theta_{H}=0^{\circ}, 30^{\circ}, 60^{\circ}, 65^{\circ}$ and $90^{\circ}$.

At the first stage of analytical calculations, we have to find the $R_{\max }$ and $R_{\min }$ from the measured data for each sample at $\theta_{H}=0^{\circ}$ and $90^{\circ}$. Note that, the demagnetising field $H_{d}$ has not been known yet but it can be calculated by using the self-consistent method. First step, we choose an initial value of demagnetising field $H_{d}$, calculate the angle $\theta$ from Eq. (5) and then fit the Eq. (3) to the experimental data of $R$ with an error $\varepsilon$. Second one, we put the angle $\theta$ back to the equation (5) to obtain the new value of $H_{d}$ and return to first step until the $\varepsilon<10^{-3}$. We have $H_{d}$ after the self-consistent process completes. In the all of figures, the measured data are presented by the dots and the solid lines are the plots of equation (4).

Figures 4(a)-4(c) show the percentage of the magnetoresistance $\Delta R / R_{\min }$ for $\theta_{H}=0^{\circ}, 30^{\circ}$, $60^{\circ}, 65^{\circ}$ and $90^{\circ}$. At zero field, the measured resistance of Ni NWs is about 800,300 and 100 for PC10, PC30 and PC100, respectively. The AMR ratio is about $1.48 \%, 2.45 \%$ and $2.71 \%$ those decrease with increasing the magnetic orientation at the magnitude $H>0$. The magnetization of Ni NWs tends to the saturation state at $\theta_{H}=90^{\circ}$ and the magnitude $H>5 \mathrm{kOe}$. The calculated curves are excellent fitting with the measured data (dotted lines) with $H_{d} \approx 2.6 \mathrm{kOe}$. The results clearly show the existence of AMR effect where the magnetoresistance strongly depends on both the magnitude and orientation of external field.

Fig. 4 also shows the single jump in the magnetoresistance through the varying field. It means that the magnetic anisotropy of the Ni NW is uniquely due to the shape anisotropy (the dipolar field or the demagnetizing field). The average of all magnetic anisotropies approximates 
to zero because the Ni NW is composed of the nanocrystallites with random orientations. The magnetic hysteresis loop confirms our samples to be the single-domain magnets $[12,14,15]$ and the valid value of $H_{d}$.
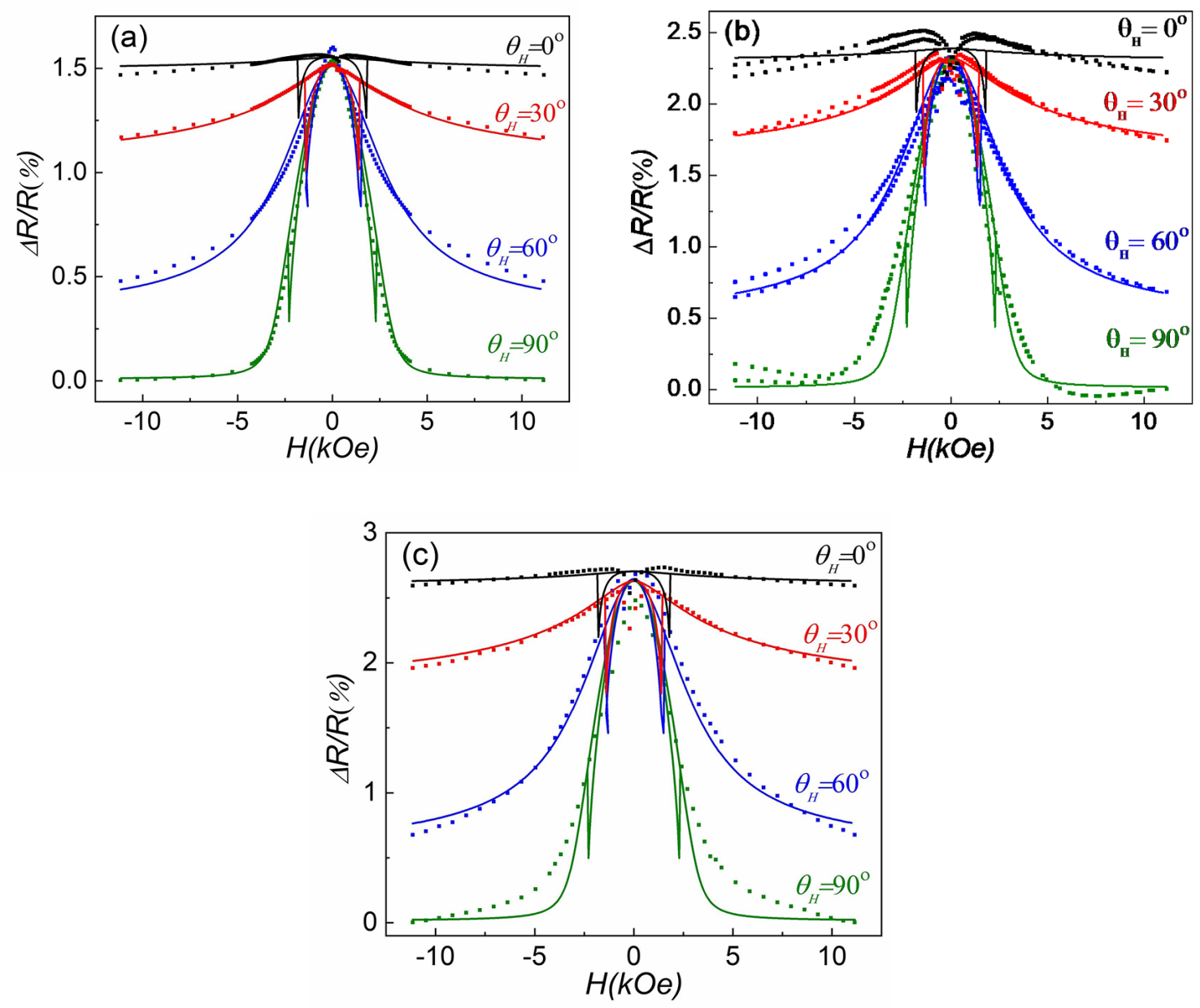

Fig. 4. The dependence of the $\Delta R / R_{\min }$ on the magnetic field $H$ with various angles $\theta_{H}$ for (a) PC10, (b) PC30 and (c) PC100 Ni NWs.

Theoretically, we plot in Fig. 5a the magnetoresistance (from Eq. (4)) for $H_{d}=2.6 \mathrm{kOe}$ and $\theta_{H}=65^{\circ}$, where the direction of applied field is parallel to the easy axis of magnetization of nickel material. In the range of $\pm H_{C} \approx 1.5 \mathrm{kOe}$, we obtained the two values of the magnetoresistance which correspond to the two solutions of equation (5) with variable $H$. The ratio $m_{z} / m_{s}=\cos ^{2} \theta$ clearly shows the hysteresis loop in Fig. 5b. Note that, the rectangle form of the hysteresis loop for $H=0$ is a signature of the single-domain magnets. 

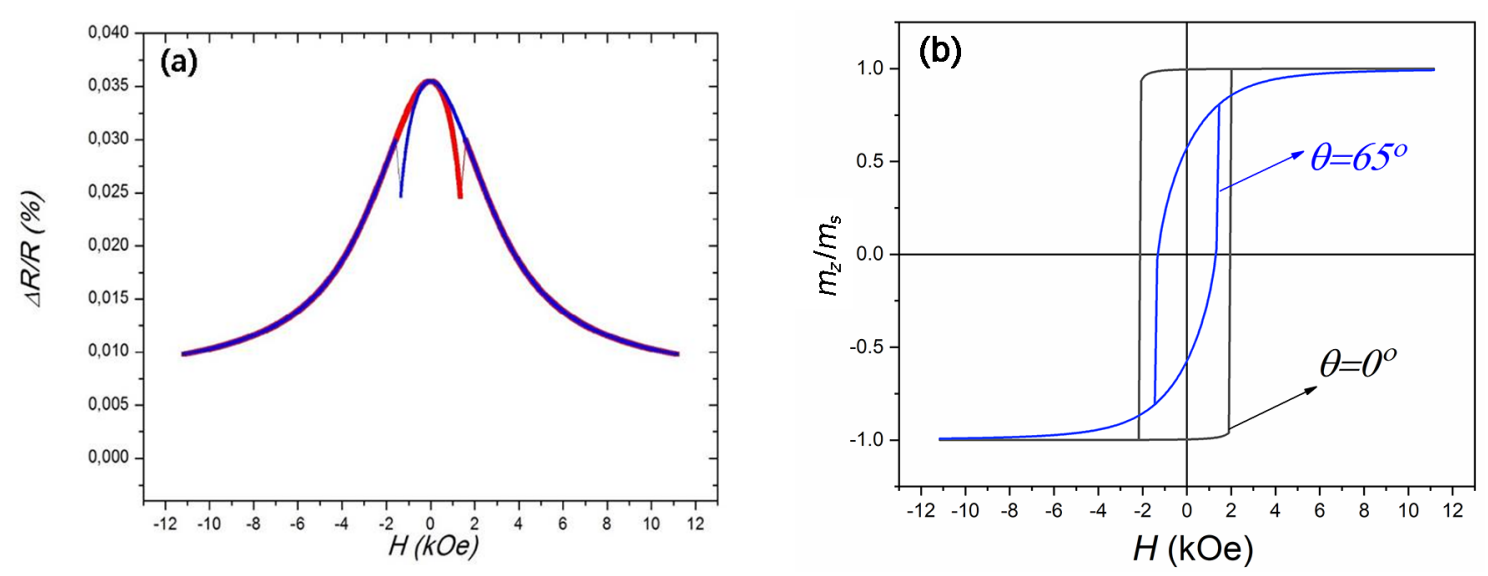

Fig. 5. The $\Delta R / R_{\min }(\mathrm{a})$ and the hysteresis loop (b) of the Ni nanowire versus the applied field with $\theta_{H}=65^{\circ}$ (blue) and $\theta_{H}=0^{\circ}$ (black).

\section{CONCLUSION}

We studied in this paper the magnetoresistance effects in the nickel nanowire of sizes 10 $\mathrm{nm}, 30 \mathrm{~nm}$ and $100 \mathrm{~nm}$. The results show that the nickel nanowires are the single-domain magnets with a unique demagnetizing field. The anisotropy of Ni NWs is as a result of the shape anisotropy only.

The experimental data are verified by analytical calculations based on the Stoner-Wohlfarth model. So, the demagnetizing field in the Ni NWs could be calculated by fitting the measured data to the expression of resistance. The combination of experiment and theoretical methods gives us an efficient approach to study the effects of anisotropy magnetoresistance in the ferromagnetic nano materials with the high precision and the low cost.

\section{ACKNOWLEDGMENT}

This work was supported by the NAFOSTED (National Foundation for Science and Technology Development, Vietnam), Grant No. 103.01-2017.62.

\section{REFERENCES}

[1] I. Žutić, J. Fabian and S. D. Sarma, Reviews of Modern Physics 76 (2004) 323.

[2] S. A. Wolf, Science 294 (2001) 1488.

[3] A. Fert and I. A. Campbell, Physical Review Letters 21 (1968) 1190.

[4] A. Fert and S.-F. Lee, Physical Review B 53 (1996) 6554.

[5] A. Fert and H. Jaffrès, Physical Review B 64 (2001) 184420.

[6] T. McGuire and R. Potter, IEEE Transactions on Magnetics 11 (1975) 1018.

[7] J.-E. Wegrowe, S. Gilbert, D. Kelly, B. Doudin and J.-P. Ansermet, IEEE Transactions on Magnetics 34 (1998) 903.

[8] J.-E. Wegrowe, D. Kelly, A. Franck, S. E. Gilbert and J.-P. Ansermet, Physical Review Letters 82 (1999) 3681.

[9] M. I. Hossain, M. Maksud, A. Subramanian, J. Atulasimha and S. Bandyopadhyay, Journal of Physics D: Applied Physics 49 (2016) 455003. 
[10] A. Fert and L. Piraux, Journal of Magnetism and Magnetic Materials 200 (1999) 338.

[11] L. Piraux, Applied Sciences 10 (2020) 1832.

[12] S. N. Kozlov, O. V. Skryabina, S. V. Egorov, I. A. Golovchanskiy, A. A. Klimenko, K. S. Napolskii and V. S. Stolyarov, Journal of Applied Physics 125 (2019) 063902.

[13] T. da Câmara Santa Clara Gomes, N. Marchal, F. A. Araujo and L. Piraux, Applied Physics Letters 115 (2019) 242402.

[14] O. V. Skryabina, S. N. Kozlov, S. V. Egorov, A. A. Klimenko, V. V. Ryazanov, S. V. Bakurskiy, M. Y. Kupriyanov, N. V. Klenov, I. I. Soloviev, A. A. Golubov, K. S. Napolskii, I. A. Golovchanskiy, D. Roditchev and V. S. Stolyarov, Scientific Reports 9 (2019) 14470.

[15] F. Zighem, D. Faurie, M. Belmeguenai, A. Garcia-Sanchez, P. Lupo and A. O. Adeyeye, Applied Physics Letters 111 (2017) 052408.

[16] W. Wernsdorfer, B. Doudin, D. Mailly, K. Hasselbach, A. Benoit, J. Meier, J. P. Ansermet and B. Barbara, Physical Review Letters 77 (1996) 1873.

[17] M. Staňo and O. Fruchart, Magnetic nanowires and nanotubes, Handbook of Magnetic Materials, Elsevier, 2018, pp. $155-267$.

[18] D.-C. Pham, N. Biziere, G. Melilli, R. Pajon, D. Lacour, L. Bouvot, M. Tabellout, D. Lairez, H.-J. Drouhin, M.-C. Clochard and J.-E. Wegrowe, Materials Research Express 1 (2014) 045017.

[19] G. Melilli, B. Madon, J.-E. Wegrowe and M.-C. Clochard, Nuclear Instruments and Methods in Physics Research Section B: Beam Interactions with Materials and Atoms 365 (2015) 675. 